\title{
Rac1 Inhibition Prevents Tissue Contraction and MMP Mediated Matrix Remodeling in the Conjunctiva
}

\author{
Victoria E. Tovell, ${ }^{1}$ Chi Y. Chau, ${ }^{1}$ Peng T. Khaw, ${ }^{2}$ and Maryse Bailly ${ }^{1}$
}

Purpose. To evaluate the efficiency of Rac1 inhibition in preventing matrix contraction by Tenon's capsule fibroblasts.

Methods. The involvement of Rac1 in serum-stimulated matrix contraction by human Tenon's fibroblasts (HTFs) was investigated in a classic collagen contraction model and our ex vivo model of tissue contraction using immunocytochemistry, chemical inhibitors, and small interfering RNA (siRNA) technology. Matrix integrity was assessed using confocal reflection microscopy and Coomassie blue staining. Quantitative real-time polymerase chain reaction (QRT-PCR) and Western blot analysis were used to assess matrix metalloproteinase (MMP) expression.

Results. Serum induced Rac1 activation in HTF-populated collagen gels and stimulated HTFs to contract collagen matrices down to $\sim 90 \%$ of their original size. Rac1 inhibition using NSC23766 or depletion using siRNA both significantly reduced HTF-mediated contraction. Early brief exposure to NSC23766 reduced HTF-mediated gel contraction by $70 \%$, while transient treatment with the Rac1 inhibitor once a week decreased ex vivo tissue contraction down to serum-free levels. Transient exposure to NSC23766 prevented early cell protrusions, fiber alignment, and matrix degradation, as seen upon continuous exposure to broad-spectrum MMP inhibitor. However, unlike MMP inhibition, transient treatment with NSC23766 led to a significant reduction in MMP1 mRNA and protein expression during contraction, without increasing MMP2 and MMP14 expression.

Conclusions. Rac1 inhibition efficiently prevents conjunctival tissue and collagen matrix contraction and prevents matrix degradation. (Invest Ophthalmol Vis Sci. 2012;53:4682-4691) DOI:10.1167/iovs.11-8577

From the Departments of ${ }^{1}$ Cell Biology and ${ }^{2}$ Ocular Biology and Therapeutics, the Moorfields Eye Hospital/UCL Institute of Ophthalmology National Institute for Health Research Biomedical Research Centre, and UCL Partners Academic Health Science Centre, London, United Kingdom.

Supported by Medical Research Council (MRC) Grant G0801049. Also supported by a grant from Fight for Sight (MB) and grants from the National Institute for Health Research Biomedical Research Centre (BMRC) at Moorfields Eye Hospital and UCL Institute of Ophthalmology, Fight for Sight, the Helen Hamlyn Trust, and Moorfields Special Trustees (PTK). Laboratories and imaging facilities were supported by the Wellcome Trust and the MRC.

Submitted for publication September 12, 2011; revised April 12 and May 11, 2012; accepted June 6, 2012.

Disclosure: V.E. Tovell, None; C.Y. Chau, None; P.T. Khaw, Alcon (C), Bausch \& Lomb (C), Pfizer (C), AstraZeneca (C), P; M. Bailly, None

Corresponding author: Victoria E. Tovell, Department of Cell Biology, UCL Institute of Ophthalmology, 11-43 Bath Street, London, EC1V 9EL, UK; v.tovell@ucl.ac.uk.
Contractile scarring following surgery or disease processes is still a major clinical challenge causing considerable morbidity. The successful outcome of glaucoma filtration surgery (GFS) is dependent upon the prevention of contractile scarring at the surgical site. Fibroblasts from the Tenon's capsule are known to play a key role in contractile scarring following GFS, or in diseases such as mucous membrane pemphigoid, by remodeling the extracellular matrix (ECM). However, signaling events that regulate matrix contraction and remodeling during wound healing are poorly understood, and the current treatment used to counteract the scarring process, Mitomycin C (MMC), can lead to cell cytotoxicity and severe side effects. ${ }^{1-3}$ Therefore, safer anti-scarring strategies, which target contraction directly, would improve clinical outcomes. The benefits of understanding the interplay between cellular signaling events and ECM remodeling during fibroblast contraction and wound healing would be very significant for ocular scarring, and also for the many medical conditions associated with contractile scarring.

Several studies have focused on MMP inhibition as a potential anti-scarring strategy. MMPs are zinc-dependent endopeptidases responsible for cleaving ECM proteins and are produced by a variety of cell types after injury. ${ }^{4-11}$ MMPs can be divided into sub-families depending on their catalytic activity or whether they are membrane bound or not. Such families include collagenases, gelatinases, and membrane-type MMPs (MT-MMP), each of which is responsible for cleaving different types of ECM proteins. A number of MMPs are associated with the GFS wound site and scarring in vivo, ${ }^{12}$ which is consistent with evidence of matrix degradation and remodeling. ${ }^{12,13}$ We have shown previously that collagen degradation and matrix remodeling are an essential part of the contraction process, both in a classic in vitro model of fibroblast-mediated collagen matrix contraction ${ }^{14}$ and in a recently developed ex vivo model of intact conjunctiva contraction. $^{13}$ In accordance with these studies, ${ }^{13,14}$ the broad-range MMP inhibitor, GM6001, has been shown to successfully inhibit HTF-mediated contraction in vitro and postoperative scarring after experimental GFS. ${ }^{15-17}$ However, the efficiency of the inhibitor is currently dependent on continuous exposure to the drug, which can trigger a reactive overexpression of some MMPs by the cells ${ }^{14,18}$ and may complicate its use in clinical applications.

Contracting fibroblasts reorganize their cytoskeletal components to generate a tensile strength, which is essential for their role in postoperative scarring. ${ }^{19}$ Small Rho GTPases are involved in regulating cytoskeletal organization, the most studied of which are Cdc42, Rac, and Rho. While Rho activation primarily stimulates stress fiber formation and focal adhesions, Cdc42 and Rac stimulate the formation of filopodia and lamellipodia, respectively. The role of Rho GTPases in actin dynamics is well documented, and it has long been understood that Rac1 can induce plasma membrane protrusions by activating actin polymerization. ${ }^{20-22}$ Rac1 activity has been shown to be essential for protrusive activity, tissue repair, and 

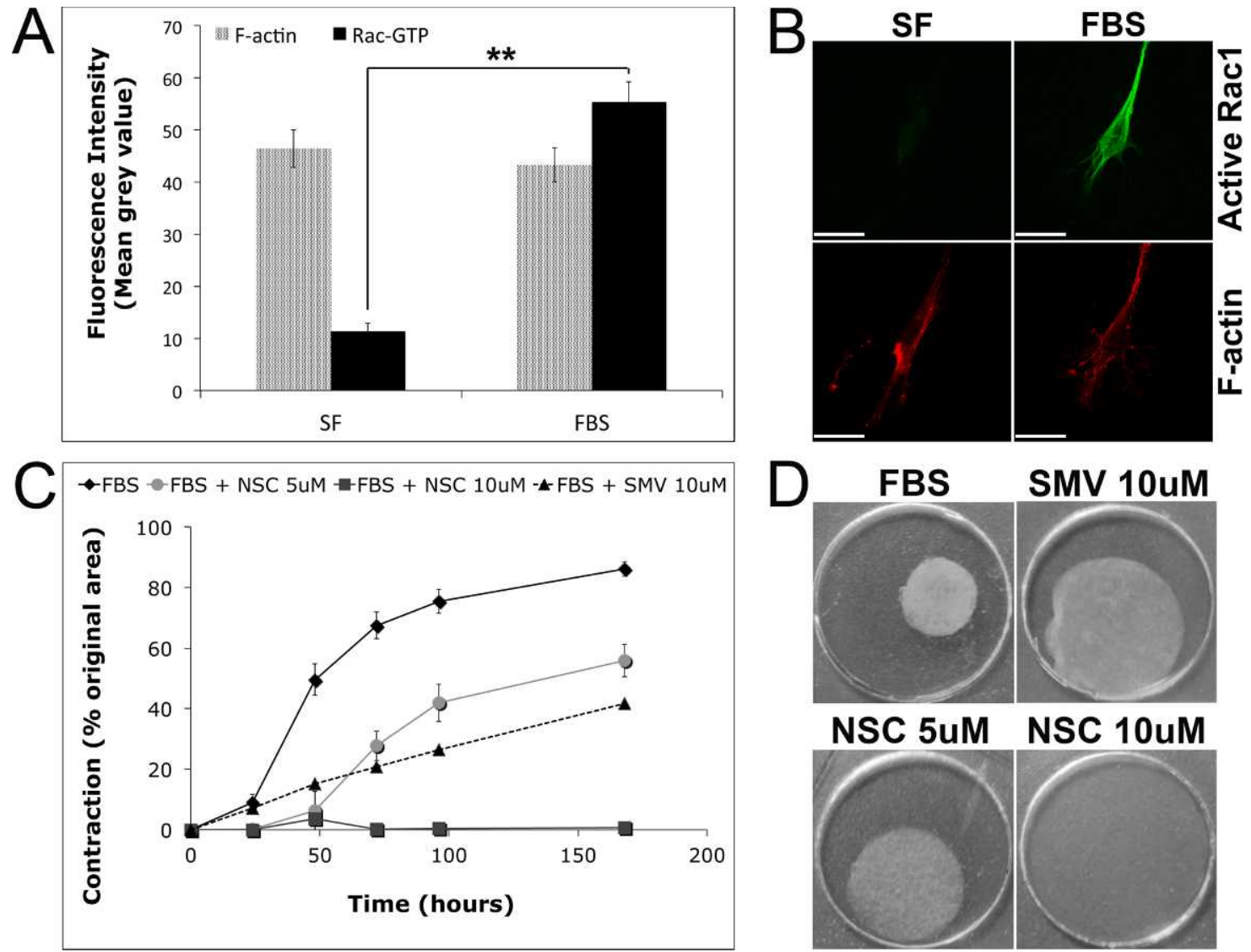

Figure 1. Serum stimulates Rac1 activation during HTF-mediated gel contraction. (A) Cells seeded in collagen matrices were serum starved for 24 hours followed by either treatment with serum (FBS) or continued serum free (SF) for 24 hours. Cells were fixed and stained for F-actin and GTPRac1, and fluorescence levels were measured on confocal sections using Image J. Results are mean \pm SEM for 3 experiments, with a total of 90 cells per treatment; ${ }^{* *} P<0.01$. (B) Representative confocal Z-stack projection of HTFs stained for active Rac1 and F-actin. Scale bar, $30 \mu \mathrm{m}$. (C) Cells seeded in collagen matrices were incubated with 10\% FBS alone (FBS) or FBS in the presence of simvastatin (SMV, $10 \mu \mathrm{M}$ ) or Rac inhibitor NSC23766 (NSC, 5 or $10 \mu \mathrm{M}$ ). Gel contraction was monitored daily. Shown are mean \pm SEM for 3 experiments, each carried out in duplicate. (D) Representative gel areas at day 7 in the presence of different inhibitors.

wound healing, ${ }^{23,24}$ and our own work has shown that protrusive activity is an essential component of early matrix contraction, suggesting that signaling through Rac1 could be one major component of contractile scarring. ${ }^{14,25}$ However, so far, no studies have looked into the role of Rac1 in Tenon's fibroblast-mediated matrix remodeling and contraction.

We thus sought to explore the potential of targeting the small GTPase Rac1 to modulate tissue contraction and scarring using both the in vitro collagen gel contraction model ${ }^{26-28}$ and our ex vivo model of conjunctival contraction. ${ }^{13}$ Using both model systems, we demonstrate here the effects of Rac1 inhibition on contraction and matrix remodeling. We reveal for the first time a connection between Rac1 and MMP activity during Tenon's fibroblast contraction and show that transient Rac1 inhibition is sufficient to significantly reduce matrix contraction, degradation, and MMP1 expression.

\section{MeTHODS}

\section{Cell Culture}

HTFs were isolated from donor tissue in accordance with the tenets of the Declaration of Helsinki and local ethics approval as previously described. ${ }^{13}$ HTFs were routinely grown in Dulbecco's modified Eagle's medium (DMEM) containing $2 \mathrm{mM}$ L-glutamine, $100 \mathrm{U} / \mathrm{mL}$ penicillin, and $100 \mu \mathrm{g} / \mathrm{mL}$ streptomycin, supplemented with $10 \%$ fetal bovine serum (FBS). The following conditions were used throughout the study: FBS (as above); granulocyte macrophage (GM) (FBS + GM6001, MMP inhibitor, $100 \mu \mathrm{M}$; Merck Chemicals Ltd., Nottingham, UK); NSC (FBS + NSC23766, Rac1 inhibitor, ${ }^{29} 50 \mu \mathrm{M}$ for the first 24 hours; Tocris Bioscience, Bristol, UK); and SMV (FBS + simvastatin, small GTPase inhibitor, ${ }^{30} 10 \mu \mathrm{M}$; Enzo Life Sciences, Exeter, UK).

\section{Collagen Gel and Ex Vivo Contraction Assays}

The collagen contraction assays were performed as previously described. ${ }^{13}$ Briefly, HTFs were seeded in a collagen type-I matrix (First Link Ltd., Birmingham, UK) at a concentration of 50,000 cells/ $\mathrm{mL}$. The gels were detached from the edge of the well, and $2 \mathrm{~mL}$ DMEM was added containing the appropriate conditions. Digital photographs were obtained daily over 7 days. Lattice areas were measured using ImageJ software (in the public domain; http://rsb.info.nih.gov/ij/), and contraction was expressed as a percentage of gel area normalized to day 0 . The ex vivo intact tissue contraction assay was performed as previously described using porcine eyes. ${ }^{13}$

\section{Microscopy and Quantification of Active Rac1 Staining}

Confocal reflection microscopy and live staining of cells in the conjunctiva fragments were performed as previously described. ${ }^{13,14}$ Immunofluorescent staining was carried out by fixing the samples with $3.7 \%$ formaldehyde (Sigma, Poole, UK) followed by a 30-minute 
incubation in $0.5 \%$ Triton-X100 (Sigma) and a 30-minute wash with 0.1 M glycine. Rhodamine-phalloidin (1:10; Invitrogen, Paisley, UK) was added to the samples for 30 minutes before they were washed, mounted, and viewed using a confocal laser scanning microscope (Zeiss Axiovert S100/Biorad Radiance 2000; Zeiss, Cambridge, UK) with a long-distance objective (LD Plan-NEOFLUAR, 63X/0.75 Korr; Zeiss). To assess active Rac1 levels in HTFs, cells were serum starved for 24 hours following gel polymerization, then treated with or without serum (FBS or SF-FBS, respectively) for 24 hours. Rac1 activity in gels was detected using anti-active (GTP)-Rac1 antibody (NewEast Biosciences, King of Prussia, PA) and by following our routine immunofluorescence protocol. ${ }^{14,31}$ Total GTP-Rac1 and F-actin levels were quantitated as integrated density levels over the whole cell area in Image J on $5-\mu \mathrm{m}$ confocal slices taken within the central area of the cells. ${ }^{31}$ To assess cell functionality following treatment with NSC23766, HTF-populated collagen gels were incubated in medium with $10 \%$ FBS and NSC23766 (50 $\mu \mathrm{M}$, NSC-FBS) or in serum-free medium (SF-FBS) for 24 hours. The medium was replaced with fresh medium with $10 \%$ FBS and the cells incubated for a further 24 hours, before being fixed and stained for Rac-GTP. Control cells were kept in serum-free medium for 48 hours (SF:SF).

\section{Measurement of Total Protein Content in Gels}

Gels were fixed in $4 \%$ formaldehyde for 30 minutes then stained in Coomassie blue for 30 minutes. The dye was extracted from the gels with $70 \%$ ethanol for 1 hour, and samples were measured for their absorbance at $550 \mathrm{~nm}$ (Fluostar Optima; BMG Biotech, Cary, NC).

\section{LIVE/DEAD Cytotoxicity Assay}

The viability of human Tenon's fibroblasts was assessed following exposure to various concentrations and application times of NSC23766 in collagen matrices. The LIVE/DEAD cytotoxicity assay (Invitrogen) was used according to manufacturer's instructions using $4 \mu \mathrm{M}$ ethidium homodimer-1 and $2 \mu \mathrm{M}$ calcein AM; live cells (green) and dead cells (red) were then visualized in gels using confocal microscopy on day 7 of the contraction assay. Z-stacks were acquired of five separate regions per gel, and two-dimensional (2-D) projections of the merged red/green images were used to count the percentage of live and dead cells.

\section{Quantitative Real-Time PCR}

Collagen gel contraction assays were ended at days $0,1,3,5$, and 7 by placing the gels straight into TRIzol Reagent (Invitrogen). Control mRNA levels at day 0 were obtained from cells in gels under serum-free conditions and lysed in TRIzol within 15 minutes of initial gel polymerization. Homogenization and phase separation were carried out according to the TRIzol manufacturer's instructions. The aqueous phase was obtained and used for RNA isolation by the Qiagen column method according to the standard protocol (RNeasy Mini Kit; Qiagen, Valencia, CA). Reverse transcription was carried out using the QuantiTect Reverse Transcription Kit (Qiagen) according to manufacturer's instructions. MMP gene expression was measured by QRT-PCR using validated primers and probes (Assay-on-Demand; Applied Biosystems, Foster City, CA). Assay identification numbers are MMP1 (Hs00899658_m1), MMP2 (Hs01548727_m1), MMP8 (Hs01029057_m1), MMP9 (Hs00234579_m1), MMP13 (Hs00233992_m1), and MMP14 (Hs00237119_m1). The HPRT1 gene was used as an endogenous control to normalize sample concentration. RT-PCR reactions were performed on an HT7900 Fast Real-Time PCR system (Applied Biosystems), and the delta analysis method ${ }^{32}$ was used for quantification of mRNA levels.

\section{Small Interfering RNA Transfection}

Rac1 was knocked down using a mixture of four SMARTselectiondesigned SiRNAs targeting human Rac1 (SiGENOME SMARTpool,

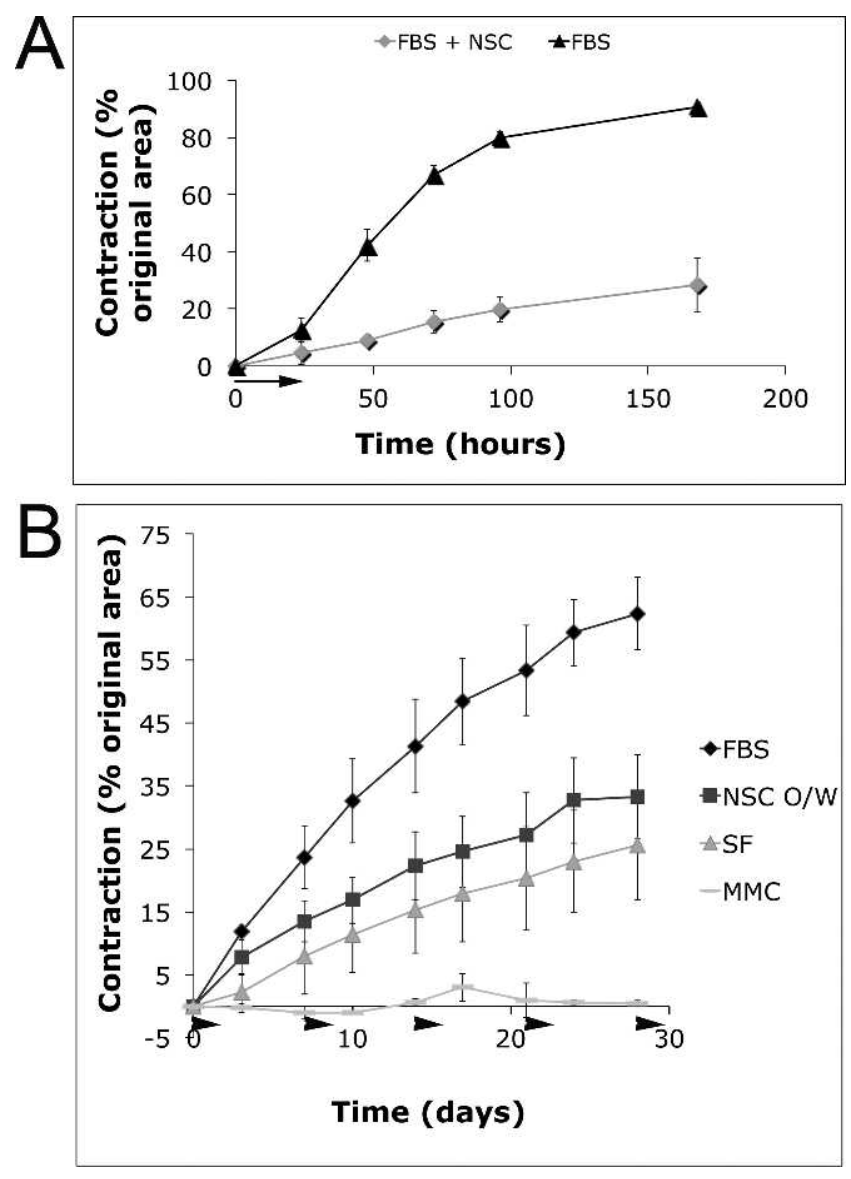

FiguRe 2. Transient treatment with the Rac1 inhibitor prevents collagen matrix contraction by HTFs and ex vivo conjunctival contraction. (A) HTF-mediated collagen gel contraction was inhibited with a single 24-hour exposure with NSC23766 (50 $\mu \mathrm{M}$; black arrow). (B) Ex vivo segments of porcine conjunctiva were used to investigate the effects of exposure to the Rac1 inhibitor in living tissue. Transient treatment with NSC23766 $(50 \mu \mathrm{M}$, for 24-hours, once a week, NSC once/week $[\mathrm{O} / \mathrm{W}]$; arrowheads) reduced FBS-mediated contraction levels down to the serum-free baseline (SF), whilst MMC $(0.5 \mathrm{mg} / \mathrm{mL}$, for 2 minutes) obliterated ex vivo live tissue contraction. Results shown are mean \pm SEM for $n=3$ experiments, each carried out in triplicate.

Darmacon) with the following target sequences: AUGAAAGUGUCACGGGUAA, CGGCACCACUGUCCCAACA, UAAAGACACGAUCGAGAAA, UAAGGAGAUUGGUGCUGUA. Cells were treated with $10 \mathrm{nM}$ Rac1 siRNA or control siRNA (SiGENOME Non-Targeting siRNA Pool No. 2) for 72 hours using HiPerFect transfection reagent (Qiagen, Lafayette, CO). Cells were then either lysed and used for Western blotting of the Rac1 protein to determine downregulation efficiency or trypsinized for the collagen gel contraction assay (as above).

\section{Western Blot Analysis}

Protein was extracted from gel samples following TRIzol manufacturer's instructions. Equal amounts of protein were loaded onto and run on $12 \%$ precast polyacrylamide mini-gels (Thermo Fisher Scientific, Northumberland, UK) and then transferred, using a semi-dry transfer system (Bio-Rad, Hercules, CA) onto polyvinylidene fluoride (PVDF) membranes (Thermo Fisher Scientific). Membranes were blocked in 3\% reduced-fat milk powder in $0.05 \%$ Tween-20 in phosphate buffered saline (TBS-T) followed by overnight incubation at $4^{\circ} \mathrm{C}$ in primary antibody (MMP1; 1:500, Abcam, Cambridge, UK or Rac1; 1:4000, BD Transduction Laboratories, Oxford, UK). Membranes were washed 3 


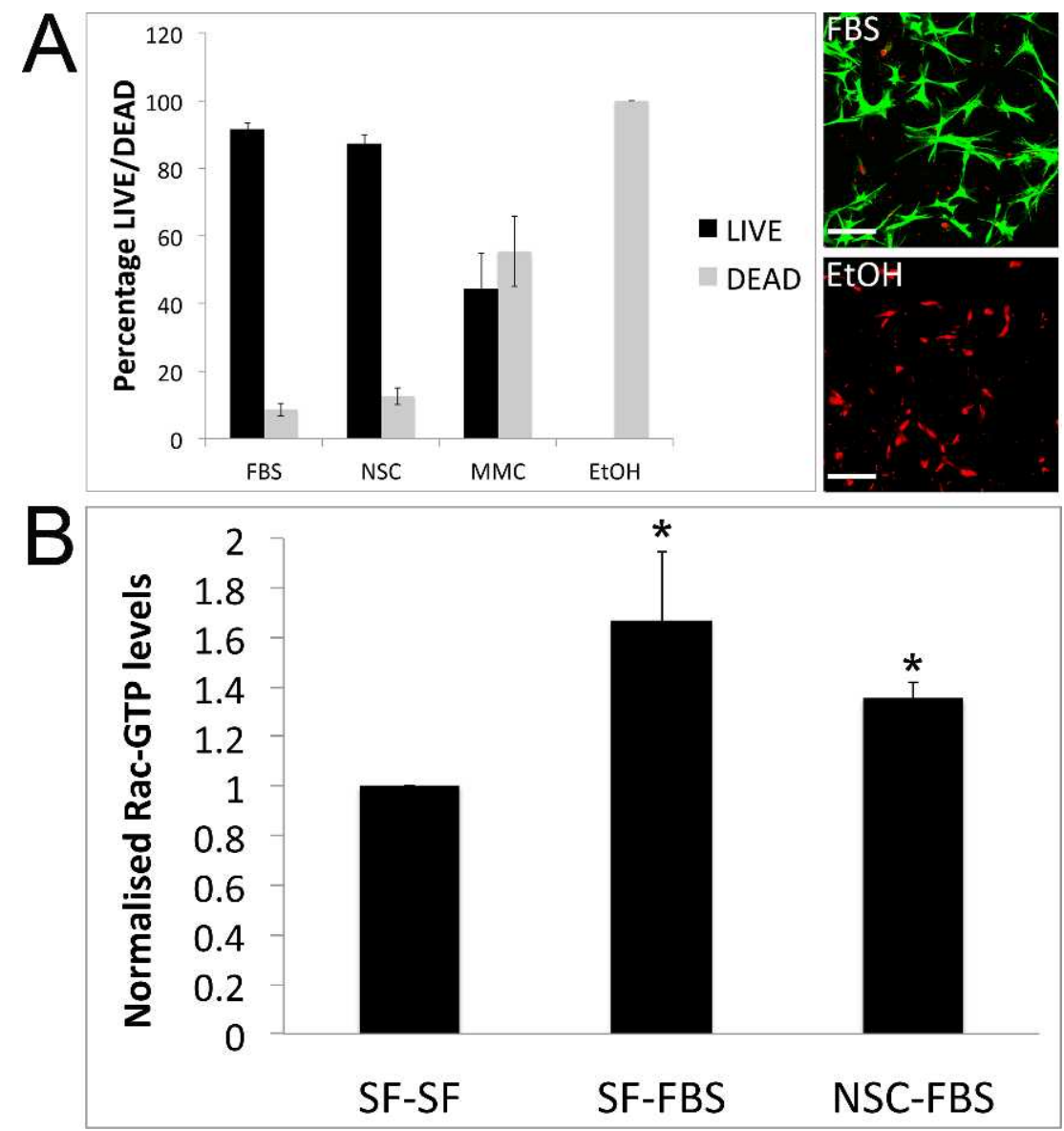

Figure 3. Transient treatment with Rac1 inhibitor is nontoxic. (A) HTFs were cast in collagen gels in the presence of $10 \%$ FBS and treated with NSC23766 (50 $\mu \mathrm{M}$, for 24 hours) or MMC $(0.2 \mathrm{mg} / \mathrm{mL}$, for 5 minutes). At day 7, a LIVE/DEAD viability assay was carried out. Live cells (green) and dead cells (red) were then visualized using confocal microscopy and quantitated within five separate regions per gel. Results shown are mean \pm SEM for $n=3$ experiments. Images show live cells in a control gel at day 7 and dead cells in a gel treated with ethanol to kill all the cells. Scale bar, $100 \mu \mathrm{m}$. (B) HTFs were cast in collagen gels in the presence of SF or NSC23766 (50 $\mu \mathrm{M})$ for 24 hours. Media was then changed to FBS for stimulation or maintained in SF for negative controls. Stimulation with FBS significantly $(P<0.05)$ increased GTP-bound Rac1 compared with SF controls even following treatment with the Rac1 inhibitor. No significant difference was found between SF-pretreated cells and NSC-pretreated cells following stimulation with serum. Results shown are mean \pm SEM for $n=4$ experiments ( 30 cells each).

times for 10 minutes in TBS-T, incubated in secondary-labeled antibody (IRDye 680, 1:5000; LI-COR, Cambridge, UK) for 2 hours at room temperature, washed, and scanned on an Odyssey IR Imager (LI-COR).

\section{Results}

\section{Serum-Mediated Matrix Contraction Is Dependent on Rac1 Activity}

Active Rac1 amounts significantly increased in HTFs within matrices following stimulation with 10\% FBS (Figs. 1A, 1B), whilst F-actin amounts remained the same. HTFs contracted collagen matrices to approximately $90 \%$ of their original size over 7 days in the presence of $10 \%$ FBS (Figs. 1C, 1D). Inhibition of Rac1 activity by continuous exposure to the Rac1 inhibitor NSC23766 (NSC) or simvastatin (SMV) significantly reduced matrix contraction by a minimum of $50 \%$. Transient treatment with the Rac1 inhibitor was also sufficient to prevent matrix contraction by $70 \%$ in collagen gels and by $50 \%$ in our ex vivo model of conjunctival contraction (Fig. 2). A single exposure with NSC23766 $(50 \mu \mathrm{M})$ for 24 hours almost fully blocked fibroblast-mediated collagen gel contraction (Fig. 2A), despite being nontoxic (Fig. 3A) and having no permanent effect on the cells' ability to activate Rac1 following serum stimulation (Fig. 3B). Similarly, application of NSC23766 (50 $\mu \mathrm{M})$ for 24 hours once a week reduced ex vivo tissue contraction to the baseline serum-free level (Fig. 2B). Nevertheless, a short exposure to MMC (Fig. 2B), equivalent to the current modalities used during GFS, ${ }^{16}$ prevented even baseline tissue contraction, owing to major cytotoxity. ${ }^{1-3}$

During GFS, a one-off treatment administrated directly to the surgical site is highly desirable. Given the effectiveness of the Rac1 inhibitor in preventing tissue and matrix contraction, we attempted to determine whether shorter incubations with higher concentrations could be as effective. Indeed, single short treatments with high concentrations of NSC23766 efficiently inhibited contraction (Fig. 4). Treating cells with $200 \mu \mathrm{M}$ NSC23766 for as little as 2 minutes was sufficient to significantly reduce contraction by HTFs, even though no toxicity was detected in concentrations up to $1 \mathrm{mM}$ for a 30 minute application.

To confirm the involvement of Rac1 in serum-mediated matrix contraction, we specifically downregulated Rac1 expression in HTFs using siRNA. HTFs treated with Rac1 siRNA displayed reproducibly lower levels of Rac1 and significantly reduced matrix contraction abilities compared with cells treated with control siRNA (Fig. 5). 


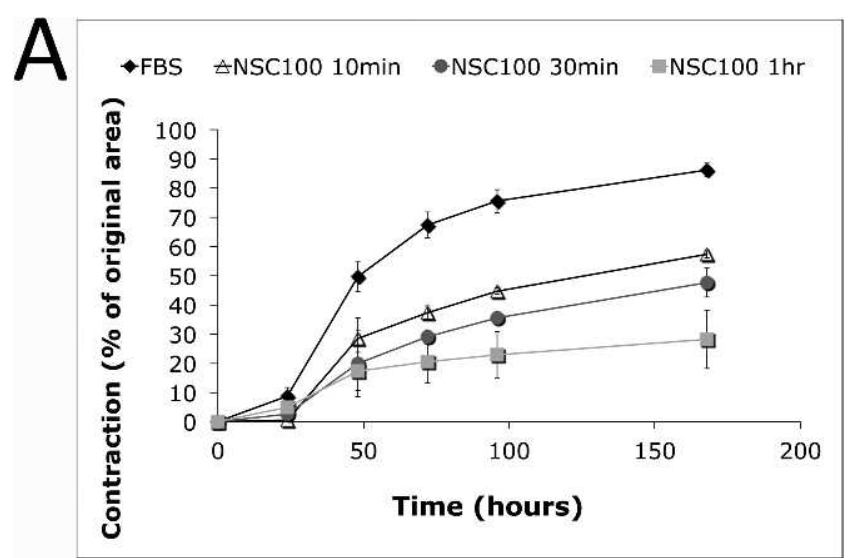

B
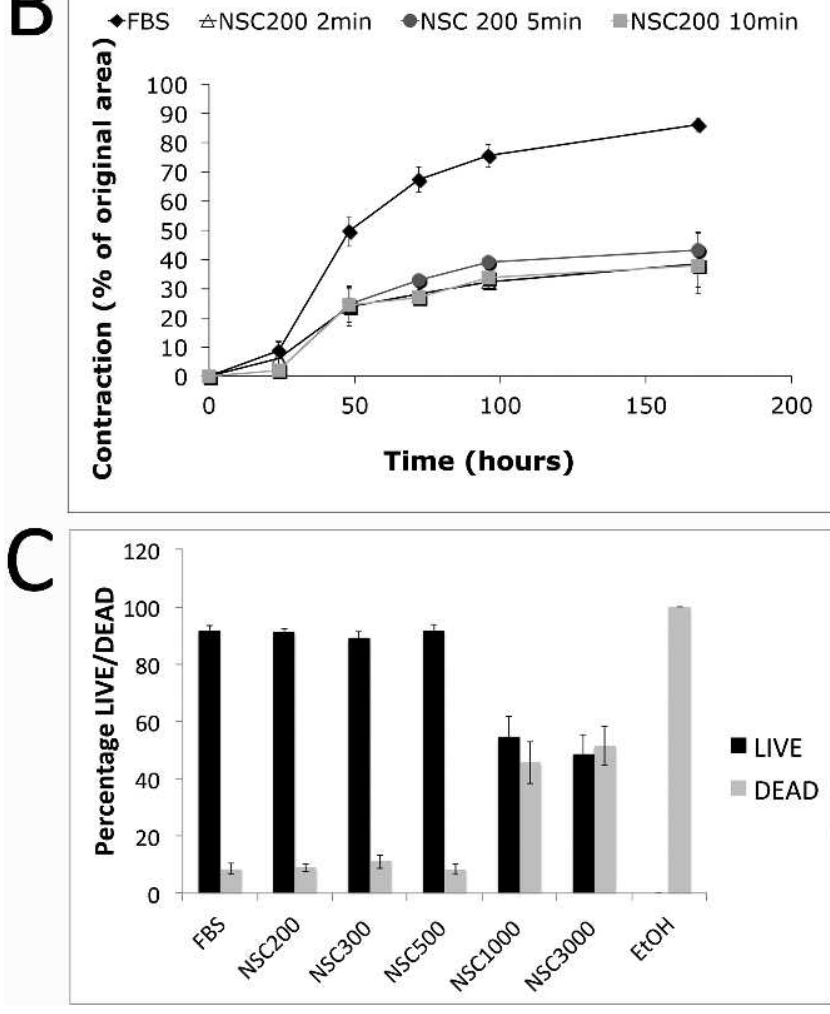

Figure 4. Short-term exposures of the Rac1 inhibitor at higher concentrations prevent HTF-mediated contraction. Tenon's fibroblasts in collagen gels were exposed to NSC23766 at (A) $100 \mu \mathrm{M}$ for 10, 30, and 60 minutes or (B) $200 \mu \mathrm{M}$ for 2, 5, and 10 minutes as soon as the gels had polymerized and then maintained in $10 \%$ serum for 7 days. (C) Cells in collagen gels were exposed to increasing concentrations of NSC23766 (200, 300, 500, 1000, $3000 \mu \mathrm{M})$ for 30 minutes and subjected to the Live/Dead assay at day 7 of the contraction assay. Significant toxicity was not detected until the millimolar range (NSC1000). Results shown are mean \pm SEM for $n=3$ experiments.

\section{Inhibition of Rac1 Signaling Prevents Cell Protrusions, Spreading, and Fiber Alignment}

Tenon's fibroblasts in collagen matrices in the presence of $10 \%$ serum for 24 hours exhibited marked processes extending from an elongated cell body (Figs. 6A, 6B). Inhibition of Rac1 altered cell spreading, with the cells exhibiting rounder cell bodies and very fine protrusions, in a manner reminiscent of that of cells in the presence of broad-spectrum MMP inhibitor GM6001 (Fig. 6). ${ }^{14}$ In addition, both inhibitors prevented the pericellular collagen fiber alignment that is normally observed in the presence of serum, ${ }^{14}$ suggesting altered cell dynamics (Fig. 6C)

\section{Inhibition of Rac1 Signaling Prevents Matrix Degradation}

Since we showed previously that matrix degradation is an essential component of matrix and tissue contraction, ${ }^{13}$ we investigated whether Rac1 inhibition affected this process. Both NSC23766 and GM6001 prevented collagen degradation in HTF-populated matrices (Fig. 7A), as measured using Coomassie blue uptake. ${ }^{13}$ Again, transient application of NSC23766 (50 $\mu \mathrm{M}$, first 24 hours) was sufficient to fully prevent matrix degradation. This effect was confirmed by confocal reflection microscopy of HTF-populated collagen matrices. Both NSC23766 and GM6001 treatment prevented degradation of collagen fibers that accompanies matrix contraction after 7 days in the presence of 10\% FBS (Fig. 7B). ${ }^{13}$

Using our ex vivo model of contraction, both tissue matrix integrity and cell viability can be visualized in live conjunctiva samples over a more physiologically relevant contraction time course. ${ }^{13}$ As shown before, fresh conjunctival segments display an abundant matrix, with a significant amount of intercalated live resident cells (Fig. $8, t=0$ ). Following 4 weeks of contraction in the presence of $10 \%$ FBS, the collagen fibers are almost completely degraded, though numerous viable cells are seen within the samples (Fig. 8, $t=28$ FBS). Treatment with MMC $(0.2 \mathrm{mg} / \mathrm{mL}$ for 5 minutes $)$ preserved fiber integrity but was extremely cytotoxic, as no live cells were visible after 28 days. In contrast, NSC23766 did not appear to affect cell viability, despite preventing matrix degradation as efficiently as MMC (Fig. 8, NCS)

\section{Transient Rac1 Inhibition Reduces MMP1 Expression}

Given the effects of the Rac1 inhibitor on matrix integrity, we tested the effect of this inhibitor on MMP expression. Collagenase mRNA expression (MMPs 1, 8, and 13) was measured in contracting Tenon's fibroblasts. MMP1 mRNA levels were significantly increased following serum stimulation compared with serum-free controls, starting at 24 hours after stimulation and remaining elevated throughout the contraction process (Fig. 9A). Expression of MMP 8 and 13 was undetectable (data not shown). Continuous treatment with GM6001 did not induce any change in MMP1 expression over the 7 days in culture. However, MMP1 mRNA expression was significantly reduced from day 5 following a single early treatment with NSC 23766 (50 $\mu \mathrm{M}$, first 24 hours), suggesting that transient treatment with the Rac1 inhibitor is sufficient to permanently alter MMP1 mRNA expression. This was confirmed at the protein level (Fig. 9B), showing a marked decrease in MMP1 expression following transient treatment with NSC23766.

The expression of other MMPs important for wound healing was also investigated, specifically the gelatinases MMP2 and MMP9, and the membrane-bound MMP14. No expression was detectable for MMP9 (data not shown), and MMP2 and MMP14 expression, though significant, did not increase during the contraction period (Fig. 10). GM6001 treatment led to a large increase in MMP2, and particularly MMP14, expression, in agreement with previous studies. ${ }^{14,18,33,34}$ On the contrary, transient treatment with NSC23766 did not increase MMP2 or MMP14 mRNA levels, with the exception of a slight increase on day 7. These data suggest that targeting Rac1 may be a potential treatment for postoperative scarring following GFS, or disease, as it prevents matrix remodeling. 

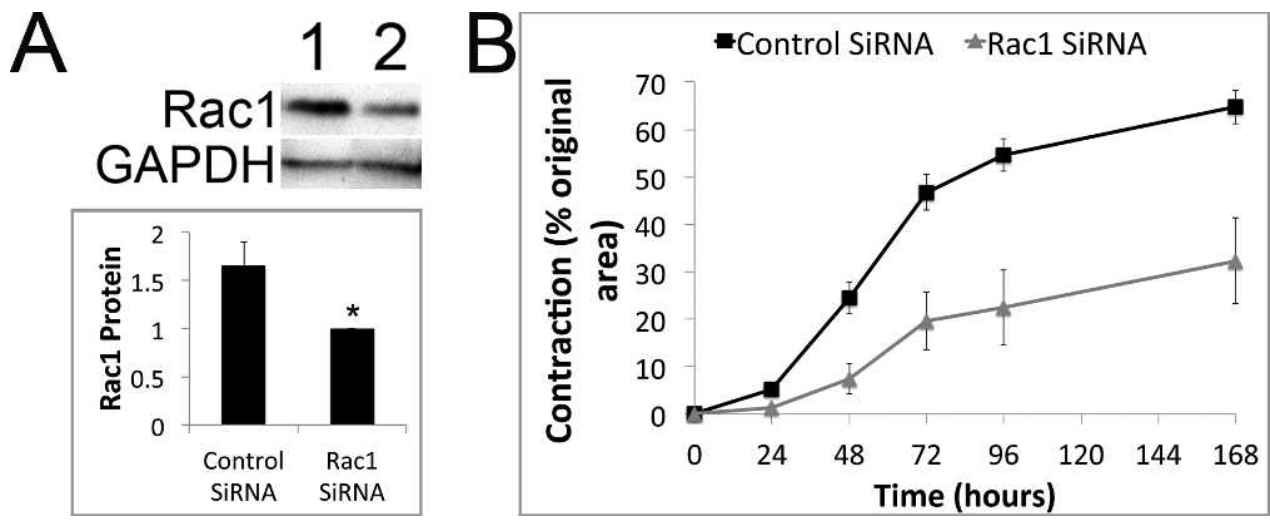

FiguRE 5. Knockdown of Rac1 in HTFs prevents collagen gel contraction (A) Rac1 SiRNA treatment led to a significant reduction in Rac1 protein expression in HTFs, ${ }^{*} P<0.05$. Shown is a representative Western blot and the corresponding quantitative protein analysis (mean \pm SEM, for $n=3$ experiments). (B) Rac1 SiRNA treatment led to a 50\% reduction in collagen gel contraction compared with SiRNA control pool. Results shown are mean \pm SEM for $n=3$ experiments, each carried out in triplicate.

\section{Discussion}

Understanding the mechanisms involved in fibroblast contraction is essential in order to establish the best therapeutic target for the prevention of postoperative scarring in the filtering bleb. We have shown previously that matrix remodeling by MMPs plays an important part in tissue contraction, both in in vitro $^{14,15}$ and in ex vivo models, ${ }^{13}$ as well as in the rabbit model of GFS. ${ }^{16,17}$ In addition, our recent work has shown that the broad-spectrum MMP inhibitor, GM6001, also has a significant effect on cell protrusive activity, which is one of the major components of early matrix contraction. ${ }^{14,25}$ The inhibitor affects both cell protrusive activity and direct collagen fiber alignment by HTFs, ${ }^{14}$ suggesting that it interferes with signal transduction pathways targeting actin dynamics. ${ }^{24}$

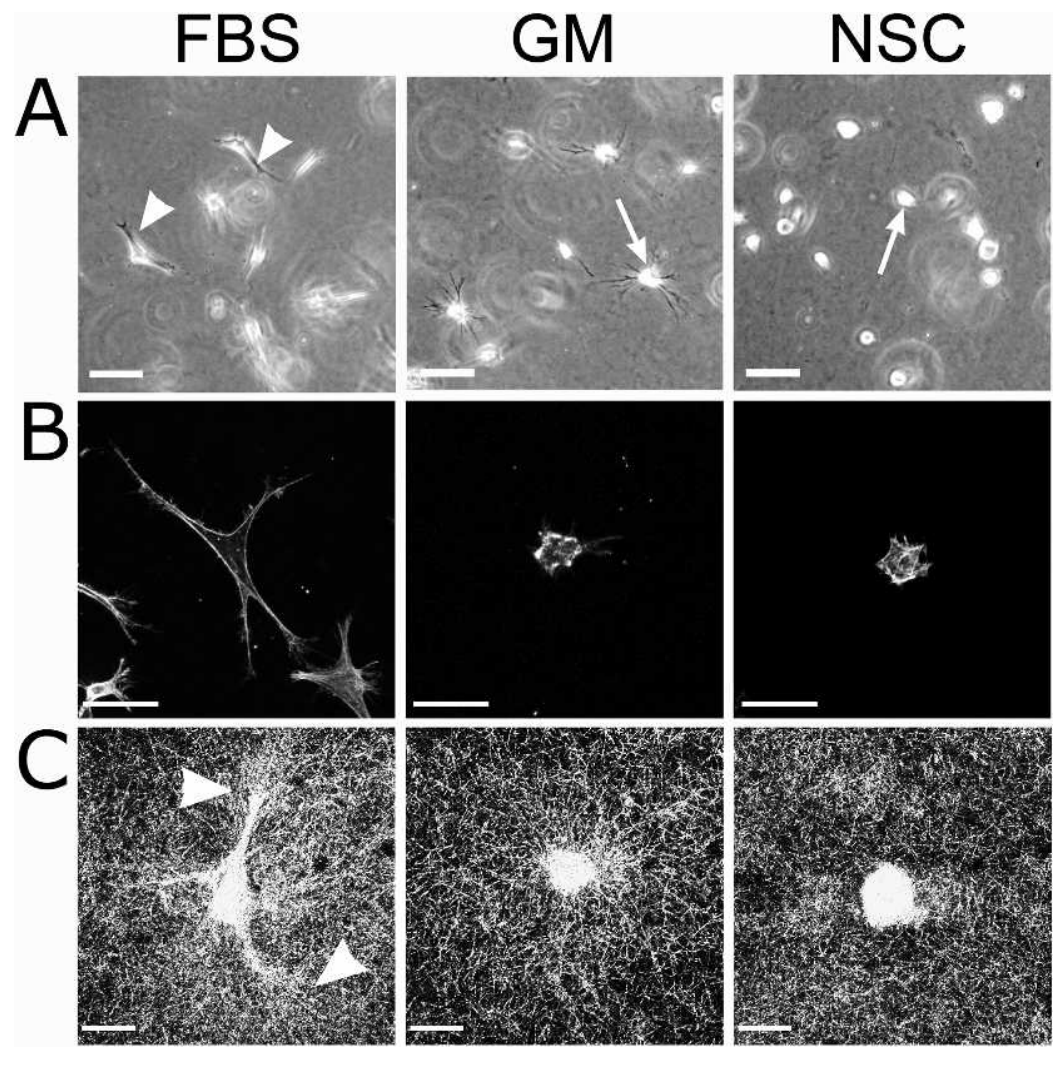

FigURE 6. Inhibition of small Rac1 or MMP activity prevents early cell protrusions, spreading, and fiber alignment. (A) Phase micrographs were taken of HTFs in 3-D collagen gels 24 hours after treatment. Cells in the presence of FBS show distinct elongated fibroblast morphologies (arrow heads). Following treatment with the MMP inhibitor GM6001 (FBS+GM, $100 \mu \mathrm{M}$ ) or the Rac inhibitor NSC23766 (FBS+NSC, $50 \mu \mathrm{M}$ ), cell morphology is altered with rounder cell bodies and finer protrusions (arrows). Scale bar, $200 \mu \mathrm{m}$. (B) F-actin (rhodamine phalloidin) staining of HTFs in collagen gels. A series of z-stacks was acquired in gels at 24 hours and merged to show a 2-D projection. Scale bar, $40 \mu \mathrm{m}$. (C) Confocal reflection microscopy showing collagen fibers in gels at 24 hours. Cells in the presence of FBS show significant fiber alignment (arrow beads), which is prevented in the presence of either the MMP inhibitor or the Rac1 inhibitor. Scale bar, $27 \mu \mathrm{m}$. 


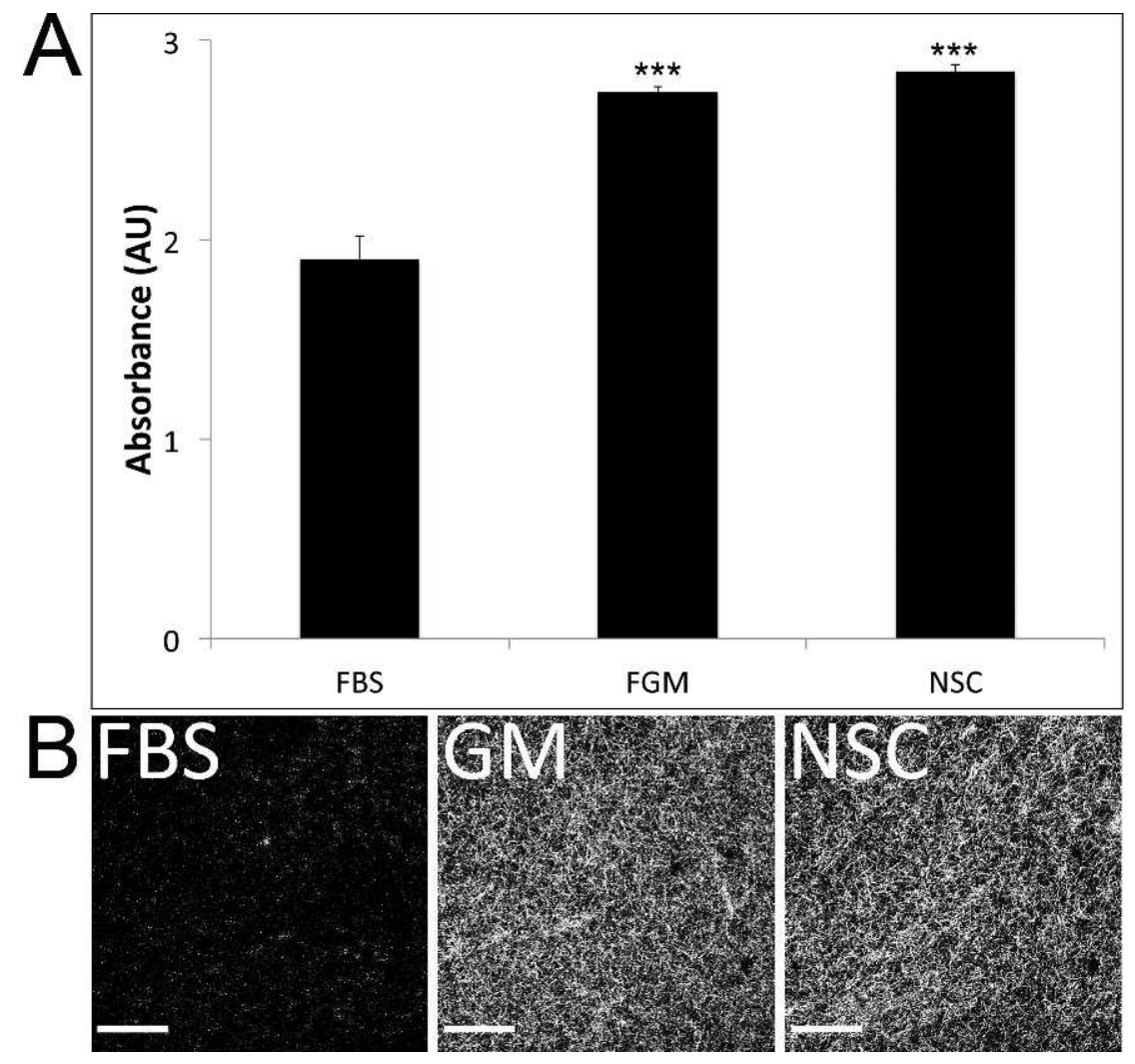

FIGURE 7. Inhibition of Rac1 signaling prevents collagen matrix degradation by HTFs. HTF cells were cast in collagen gels in the presence of $10 \%$

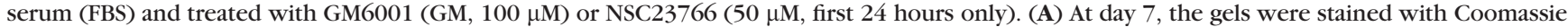
blue, and the absorbance of the extracted dye was measured at $550 \mathrm{~nm}$ to assess the total protein content, which correlates with collagen concentration. Results shown are mean \pm SEM for $n=3$ experiments. Significantly higher concentrations $(* * * * 0.001)$ in collagen are seen at day 7 following all treatments. (B) At day 7, confocal reflection microscopy was carried out to assess fiber integrity. Representative single z-sections were captured for each treatment using a cell-free area. Reflection of fibers can clearly be seen following all treatments in comparison with a lack of fibers in samples treated with FBS alone. Scale bar, $40 \mu \mathrm{m}$.

The small GTPases of the Rho family are the main regulators of the actin cytoskeleton ${ }^{22,24}$; and within this family, the small GTPase Rac1 is thought to be a master regulator of cell protrusive activity and motility. $22,24,35$ More recently, Rac1 expression has been associated with matrix remodeling in fibroblasts, both in the context of tumor-promoting stroma, ${ }^{36}$ as well as fibrotic diseases. ${ }^{37}$ Indeed, fibroblasts expressing constitutively active GTPases successfully contract collagen matrices. ${ }^{38}$ Rac1 inhibition also reverses the elevated contractile phenotype of fibroblasts from patients who suffer from the chronic fibrotic disease, scleroderma ${ }^{37}$ and alters the contractile and tumor-promoting properties of cancer-associated fibroblasts. ${ }^{36}$ Furthermore, Rac1 depletion leads to delayed wound healing in mice, with a significantly reduced contractile and fibrotic phenotype. ${ }^{39}$ We demonstrate here that Rac1 inhibition dramatically reduces matrix contraction by Tenon's fibroblasts, as well as ex vivo tissue contraction, suggesting an essential role for Rac1 in ocular scarring. Furthermore, we show that transient treatment with the Rac1 inhibitor is sufficient to prevent matrix remodeling and tissue contraction, suggesting that it can permanently alter the contractile properties of ocular fibroblasts, similar to its effect in dermal fibroblasts. ${ }^{37}$

Although inhibition of Rac1 would dramatically alter cell protrusive activity, ${ }^{22,24}$ this effect is likely to be limited to early contraction, and we have shown that full matrix contraction is linked to MMP-mediated collagen degradation and matrix remodeling. ${ }^{14,25}$ Rather, Rac1 inhibition appears to trigger changes in gene expression that prevents the cells from expressing the fibrotic phenotype, therefore blocking their ability to remodel the matrix. We show that while treatment with the broad-spectrum MMP inhibitor does not reduce the level of MMP expression, a single 24-hour exposure to the Rac1 inhibitor reduces expression of MMP1 after 3 days. While MMP inhibitors have shown great promise in modulating tissue contraction and scarring in various models, ${ }^{4,15-18}$ one of their main drawbacks is that they can lead to a significant increase in MMP expression, such as MT1-MMP in ocular and dermal fibroblasts ${ }^{14,18}$ or MMP9 in cutaneous wounds. ${ }^{33,34}$ This could lead to failure in preventing contraction in the long term ${ }^{33,34}$ if active concentrations of MMP inhibitors are not maintained. By contrast, we demonstrate here that a transient treatment with the Rac1 inhibitor prevents matrix degradation and remodeling as efficiently as continuous exposure to GM6001. However, unlike treatment with GM6001, Rac1 inhibition results in a significant long-term reduction in MMP1 expression in the later stage of contraction and does not trigger a reactive overexpression of other major MMPs. At present, it is still unclear how Rac1 regulates MMP expression, but a number of studies have shown that Rac1 inhibition results in a decrease in MMP expression, particularly MMP1, ${ }^{40-42}$ possibly through the ERK $^{41,43}$ or NFKB pathways. ${ }^{40,44}$ Further work will be needed to identify the pathway at work in HTFs as well as the full array of pro-fibrotic genes downstream of Rac1 activation.

In summary, this study shows for the first time that Rac1 is an important component of Tenon's capsule tissue contraction and that transient treatment with the Rac1 inhibitor is sufficient to prevent in vitro collagen matrix contraction, 


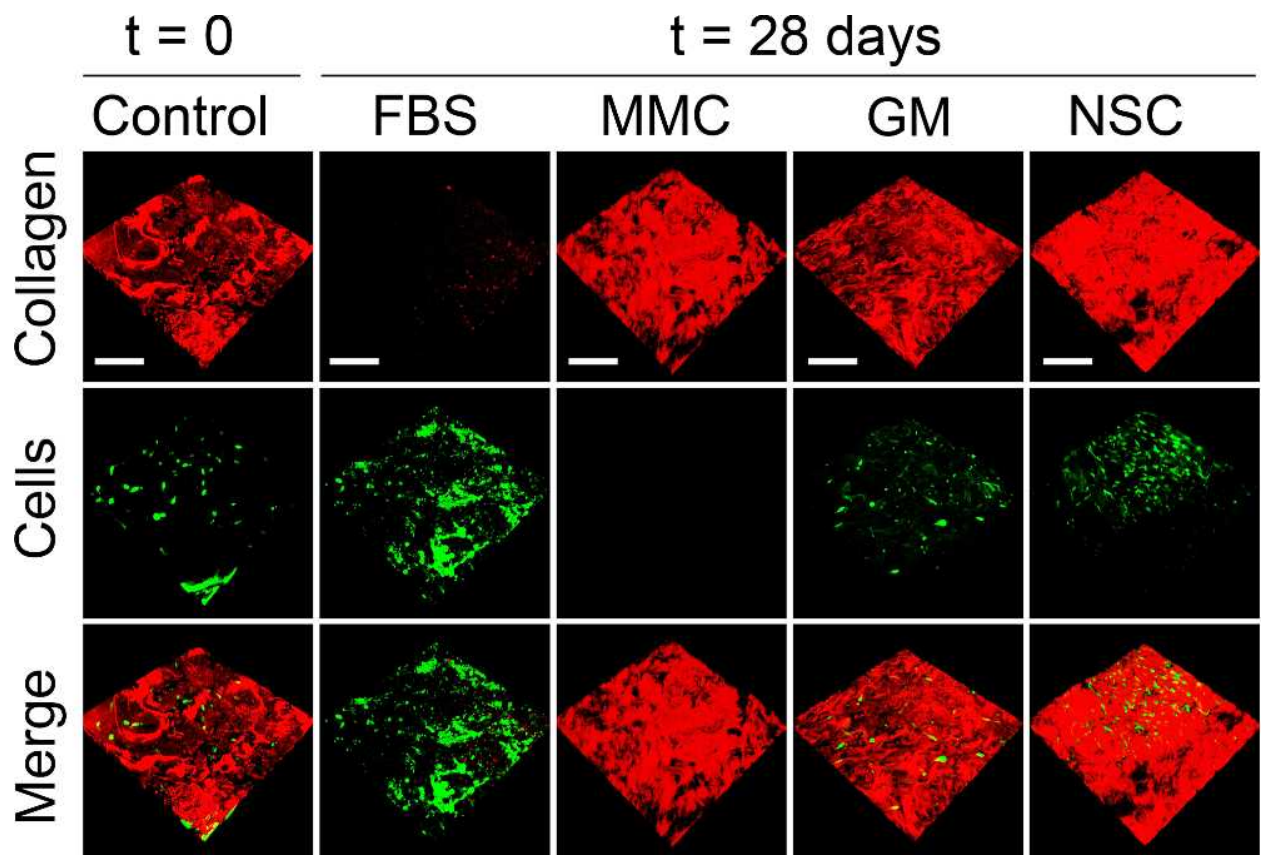

FiguRE 8. Inhibition of Rac1 signaling prevents matrix degradation in live segments of conjunctiva. Reflection microscopy of collagen fibers (red) and living cells stained with the Vibrant CFDA Cell Tracer kit (green) were visualized with confocal microscopy. Shown are full 3-D reconstructions of representative confocal z-stacks. In freshly isolated segments of porcine conjunctiva, thick collagen fibers can be seen (control, $t=0$ ). After 28 days in the presence of $10 \%$ serum (FBS), most of the collagen fibers have been degraded; however, the cells remain fully viable. MMC treatment $(0.5$ $\mathrm{mg} / \mathrm{mL}$, for 2 minutes at the onset of assay) rescues the fiber degradation induced by FBS, although no viable cells can be found. Treatment with NSC23766 (50 $\mu \mathrm{M}$ for 24 hours, once a week) also rescues fiber integrity without causing cell death. Scale bar, $90 \mu \mathrm{m}$.
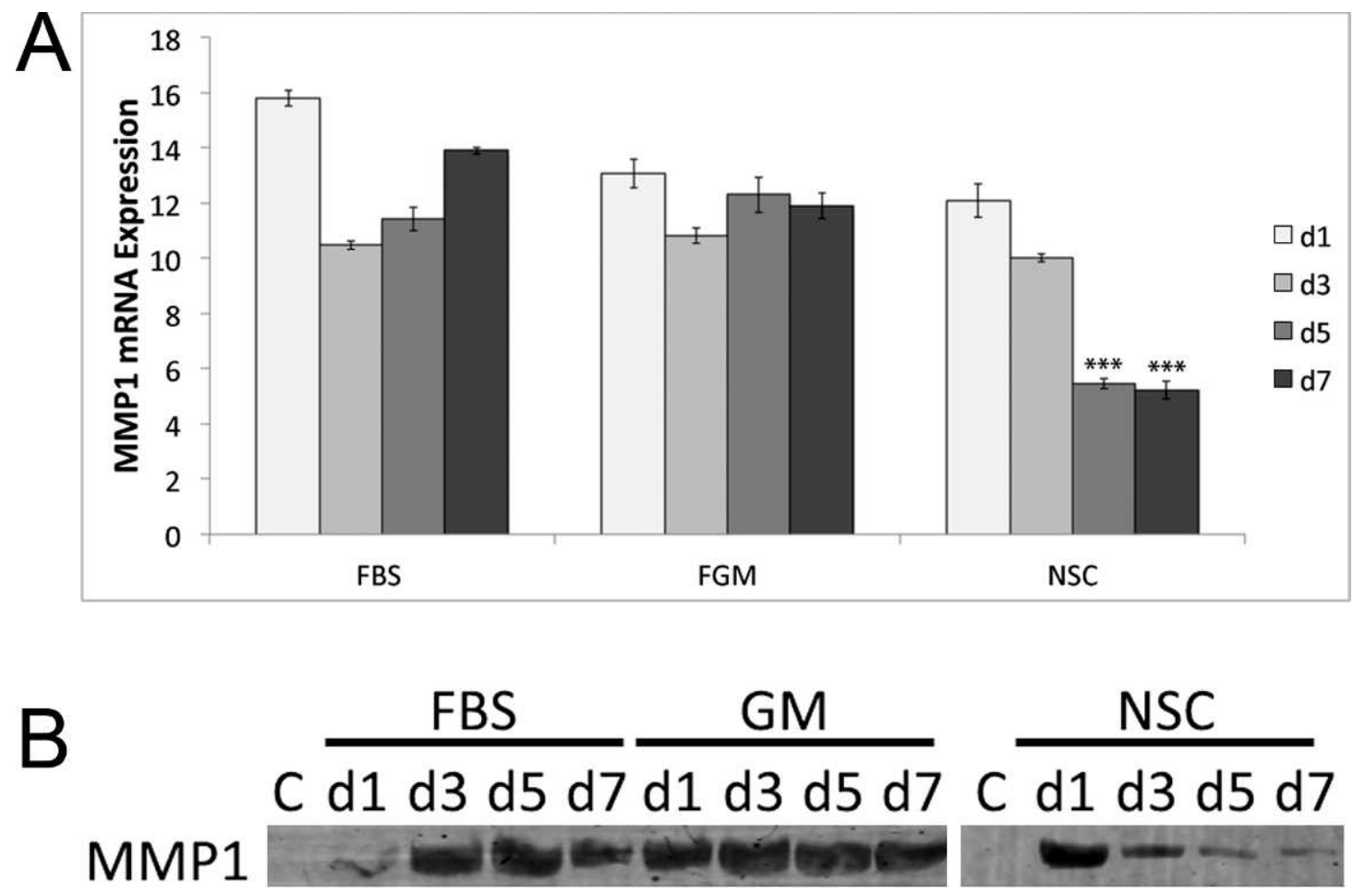

Figure 9. Transient early inhibition of Rac1 leads to a long-term reduction in MMP1 expression. MMP1 expression was investigated in contracting fibroblasts in collagen gels at days1, 3, 5, and 7, following treatment with GM6001 (100 $\mu \mathrm{M}$ continuous) or NSC23766 (50 $\mu$ M, first 24 hours only). (A) QRT-PCR results showing a significant reduction in MMP1 mRNA expression following transient treatment with NSC23766. Treatment with GM6001 caused no reduction in MMP1 gene expression. HPRT1 mRNA levels were used to normalize samples for cell numbers and individual mRNA levels are shown normalized to nontreated cells in freshly polymerized gels $(t=0)$. Significant differences are expressed as ${ }^{*} P<0.05$, ${ }^{* * *} P<0.01,{ }^{* * * *} P$ $<0.001$. Results shown are mean \pm SEM for $n=3$ experiments. (B) Western blot analysis showing a reduction of MMP1 protein expression following transient treatment with $\operatorname{NSC23766~}(n=2$, representative blot). 

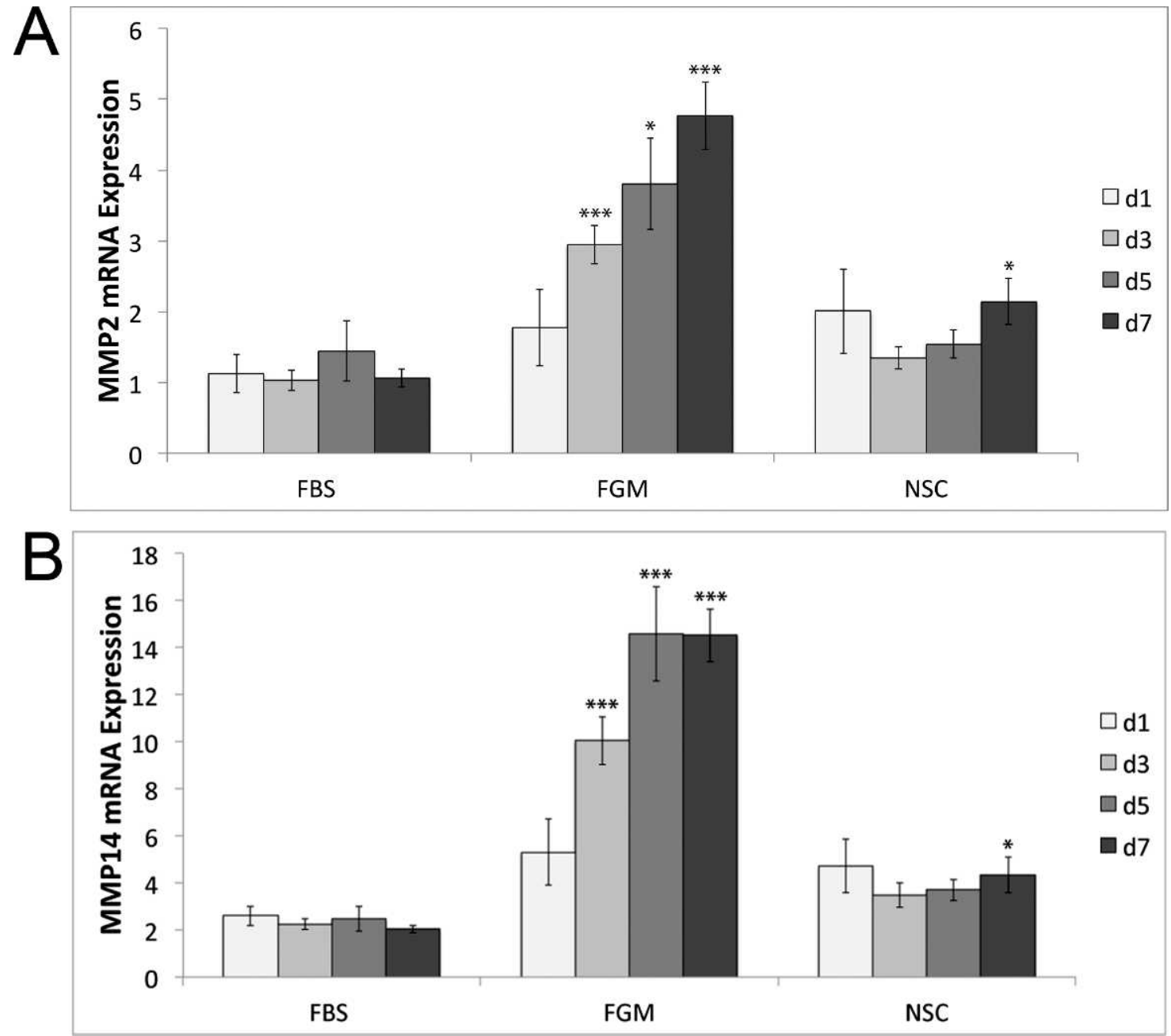

Figure 10. Transient early inhibition of Rac1 does not alter the expression of MMP2 and MMP14. QRT-PCR was used to investigate (A) MMP2 and (B) MMP14 expression in contracting fibroblasts in collagen gels at days 1, 3, 5, and 7, following treatment with GM6001 (100 $\mu$ M continuous) or NSC23766 (50 $\mu \mathrm{M}$, first 24 hours only). Treatment with GM6001 leads to an increase in MMP2 and MMP14 mRNA expression by day 3, whereas transient treatment with NSC23766 only significantly alters expression by day 7. HPRT1 mRNA levels were used to normalize samples for cell numbers, and individual mRNA levels are shown normalized to nontreated cells in freshly polymerized gels $(t=0)$. Significant differences are expressed as ${ }^{*} P<0.05,{ }^{* *} P<0.01,{ }^{* * * *} P<0.001$. Results shown are mean \pm SEM for $n=3$ experiments.

intact tissue contraction, and matrix degradation and also to reduce MMP1 expression, whilst showing no increase in mRNA expression of other MMPs.

\section{Acknowledgments}

The authors thank Janine M. Wilkinson for kindly helping with RNA isolation from cells in 3-D collagen matrices.

\section{References}

1. Jampel HD. Effect of brief exposure to mitomycin $C$ on viability and proliferation of cultured human Tenon's capsule fibroblasts. Ophthalmology. 1992;99:1471-1476.

2. Parrish R, Minckler D. "Late endophthalmitis"-filtering surgery time bomb? Ophthalmology. 1996;103:1167-1168.

3. Stamper RL, McMenemy MG, Lieberman MF. Hypotonous maculopathy after trabeculectomy with subconjunctival 5fluorouracil. Am J Ophthalmol. 1992;114:544-553.

4. Ravanti L, Kahari VM. Matrix metalloproteinases in wound repair (review). Int J Mol Med. 2000;6:391-407.
5. Daniels JT, Geerling G, Alexander RA, Murphy G, Khaw PT, Saarialho-Kere U. Temporal and spatial expression of matrix metalloproteinases during wound healing of human corneal tissue. Exp Eye Res. 2003;77:653-664.

6. Gawronska-Kozak B. Scarless skin wound healing in FOXN1 deficient (nude) mice is associated with distinctive matrix metalloproteinase expression. Matrix Biol. 2011;30:290-300.

7. Gillard JA, Reed MW, Buttle D, Cross SS, Brown NJ. Matrix metalloproteinase activity and immunohistochemical profile of matrix metalloproteinase- 2 and -9 and tissue inhibitor of metalloproteinase-1 during human dermal wound healing. Wound Repair Regen. 2004;12:295-304.

8. Hayden DM, Forsyth C, Keshavarzian A. The role of matrix metalloproteinases in intestinal epithelial wound healing during normal and inflammatory states. J Surg Res. 2011; 168:315-324.

9. Salo T, Makela M, Kylmaniemi M, Autio-Harmainen H, Larjava H. Expression of matrix metalloproteinase-2 and -9 during early human wound healing. Lab Invest. 1994;70:176-182.

10. Ye HQ, Maeda M, Yu FS, Azar DT. Differential expression of MT1-MMP (MMP-14) and collagenase III (MMP-13) genes in normal and wounded rat corneas. Invest Ophthalmol Vis Sci. 2000;41:2894-2899. 
11. Zhang H, Chang M, Hansen CN, Basso DM, Noble-Haeusslein LJ. Role of matrix metalloproteinases and therapeutic benefits of their inhibition in spinal cord injury. Neurotberapeutics. 2011;8:206-220.

12. Shima I, Katsuda S, Ueda Y, Takahashi N, Sasaki H. Expression of matrix metalloproteinases in wound healing after glaucoma filtration surgery in rabbits. Ophthalmic Res. 2007;39:315324.

13. Tovell VE, Dahlmann-Noor AH, Khaw PT, Bailly M. Advancing the treatment of conjunctival scarring: a novel ex vivo model. Arch Ophthalmol. 2011;129:619-627.

14. Martin-Martin B, Tovell V, Dahlmann-Noor AH, Khaw PT, Bailly M. The effect of MMP inhibitor GM6001 on early fibroblastmediated collagen matrix contraction is correlated to a decrease in cell protrusive activity. Eur J Cell Biol. 2011;90:26-36.

15. Daniels JT, Cambrey AD, Occleston NL, et al. Matrix metalloproteinase inhibition modulates fibroblast-mediated matrix contraction and collagen production in vitro. Invest Ophthalmol Vis Sci. 2003;44:1104-1110.

16. Wong TTL, Mead AL, Khaw PT. Long term modulation of ocular scarring by matrix metalloproteinase inhibitioncomparison with mitomycin-C treatment following experimental glaucoma filtration. Invest Ophthalmol Vis Sci. 2003; 44:U491-U491.

17. Wong TTL, Mead AL, Khaw PT. Matrix metalloproteinase inhibition modulates postoperative scarring after experimental glaucoma filtration surgery. Invest Ophthalmol Vis Sci. 2003;44:1097-1103.

18. Townley WA, Cambrey AD, Khaw PT, Grobbelaar AO. Matrix metalloproteinase inhibition reduces contraction by dupuytren fibroblasts. J Hand Surg Am. 2008;33:1608-1616.

19. Ehrlich HP, Rajaratnam JB. Cell locomotion forces versus cell contraction forces for collagen lattice contraction: an in vitro model of wound contraction. Tissue Cell. 1990;22:407-417.

20. Etienne-Manneville S, Hall A. Rho GTPases in cell biology. Nature. 2002;420:629-635.

21. Hall A. Rho GTPases and the actin cytoskeleton. Science. 1998; 279:509-514.

22. Ridley AJ. Rho GTPases and actin dynamics in membrane protrusions and vesicle trafficking. Trends Cell Biol. 2006;16: 522-529.

23. Liu SX, Kapoor M, Xu SW, Kennedy L, Abraham DJ, Leask A. Rac1 is required for tissue repair, dermal homeostasis and fibrogenic responses in vivo. Wound Repair Regen. 2008;16: A43-A43.

24. Nobes CD, Hall A. Rho GTPases control polarity, protrusion, and adhesion during cell movement. J Cell Biol. 1999;144: 1235-1244.

25. Dahlmann-Noor AH, Martin-Martin B, Eastwood M, Khaw PT, Bailly M. Dynamic protrusive cell behaviour generates force and drives early matrix contraction by fibroblasts. Exp Cell Res. 2007;313:4158-4169.

26. Bell E, Ivarsson B, Merrill C. Production of a tissue-like structure by contraction of collagen lattices by human fibroblasts of different proliferative potential in vitro. Proc Natl Acad Sci U S A. 1979;76:1274-1278.

27. Grinnell F. Fibroblasts, myofibroblasts, and wound contraction. J Cell Biol. 1994;124:401-404.

28. Occleston NL, Alexander RA, Mazure A, Larkin G, Khaw PT. Effects of single exposures to antiproliferative agents on ocular fibroblast-mediated collagen contraction. Invest Ophthalmol Vis Sci. 1994;35:3681-3690.

29. Gao Y, Dickerson JB, Guo F, Zheng J, Zheng Y. Rational design and characterization of a Rac GTPase-specific small molecule inhibitor. Proc Natl Acad Sci U S A. 2004;101:7618-7623.

30. Miller T, Yang F, Wise CE, et al. Simvastatin stimulates apoptosis in cholangiocarcinoma by inhibition of Rac1 activity. Dig Liver Dis. 2011;43:395-403.

31. McGee KM, Vartiainen MK, Khaw PT, Treisman R, Bailly M. Nuclear transport of the serum response factor coactivator MRTF-A is downregulated at tensional homeostasis. EMBO Rep. 2011;12:963-970.

32. Livak KJ, Schmittgen TD. Analysis of relative gene expression data using real-time quantitative PCR and the 2(-Delta Delta C(T)) Method. Methods. 2001;25:402-408.

33. Lund LR, Romer J, Bugge TH, et al. Functional overlap between two classes of matrix-degrading proteases in wound healing. ЕMBO J. 1999;18:4645-4656.

34. Mirastschijski U, Schnabel R, Claes J, et al. Matrix metalloproteinase inhibition delays wound healing and blocks the latent transforming growth factor-beta1-promoted myofibroblast formation and function. Wound Repair Regen. 2010;18: 223-234.

35. Yamazaki D, Kurisu S, Takenawa T. Involvement of Rac and Rho signaling in cancer cell motility in 3D substrates. Oncogene. 2009;28:1570-1583.

36. Hooper S, Gaggioli C, Sahai E. A chemical biology screen reveals a role for Rab21-mediated control of actomyosin contractility in fibroblast-driven cancer invasion. $\mathrm{Br} \mathrm{J}$ Cancer. 2010;102:392-402.

37. Shi-wen X, Liu SX, Eastwood M, et al. Rac inhibition reverses the phenotype of fibrotic fibroblasts. Plos One. 2009;4:e7438.

38. Zhang ZG, Lambert $\mathrm{C}$, Servotte $S$, et al. Effects of constitutively active GTPases on fibroblast behavior. Cell Mol Life Sci. 2006; 63:82-91.

39. Liu S, Kapoor M, Leask A. Rac1 expression by fibroblasts is required for tissue repair in vivo. Am J Pathol. 2009;174: 1847-1856.

40. Balasubramanian S, Fan M, Messmer-Blust AF, et al. The interferon-gamma-induced GTPase, mGBP-2, inhibits tumor necrosis factor alpha (TNF-alpha) induction of matrix metalloproteinase-9 (MMP-9) by inhibiting NF-kappaB and Rac protein. J Biol Chem. 2011;286:20054-20064.

41. Deroanne CF, Hamelryckx D, Ho TTG, et al. Cdc42 downregulates MMP-1 expression by inhibiting the ERK1/2 pathway. $J$ Cell Sci. 2005;118:1173-1183.

42. Igata $\mathrm{T}$, Jinnin $\mathrm{M}$, Makino $\mathrm{T}$, et al. Up-regulated type I collagen expression by the inhibition of Rac1 signaling pathway in human dermal fibroblasts. Biochem Biophys Res Commun. 2010;393:101-105.

43. Bartolome RA, Ferreiro S, Miquilena-Colina ME, et al. The chemokine receptor CXCR 4 and the metalloproteinase MT1MMP are mutually required during melanoma metastasis to lungs. Am J Path. 2009;174:602-612.

44. Venkatesan B, Valente AJ, Prabhu SD, Shanmugam P, Delafontaine P, Chandrasekar B. EMMPRIN activates multiple transcription factors in cardiomyocytes, and induces interleukin18 expression via Rac1-dependent PI3K/Akt/IKK/NF-kappa B and MKK 7/JNK/AP-1 signaling. J Mol Cell Cardiol. 2010;49: 655-663. 